\title{
High-resolution scheme based on the undetermined coefficient method and its application
}

\author{
Teng $\mathbf{W U}^{1, *}$ and Lingli $\mathrm{WU}^{2}$ \\ ${ }^{1}$ College of Harbor, Coastal and Offshore Engineering, Hohai University, Nanjing 210098, China \\ ${ }^{2}$ College of Harbor, Coastal and Offshore Engineering, Hohai University, Nanjing 210098, China
}

Received 15 May 2013; Accepted 25 July 2013

\begin{abstract}
The upwind scheme exhibits spurious oscillations in resolving the convectively dominated problems. In this paper, a high-resolution scheme for advection equation was developed by using the undetermined coefficient method to reduce the numerical diffusion. The new scheme is applied to a rectangular wave and a Gaussian wave. The results show that the new scheme agrees well with the exact solution for pure convection of a Gauss wave and the rectangular wave. The new scheme has better accuracy than the conventional upwind scheme.
\end{abstract}

Keywords: advection equation, undetermined coefficient method, numerical scheme, upwind scheme

\section{Introduction}

The advection-diffusion equation has been widely used to simulate sediment transport, pollutant advection and heat transfer, and so on. But it is hard to get the exact solution for the advection-diffusion equation [1,2,3]. Therefore, many numerical schemes have been proposed to get the numerical solution of the equation. Advective transport refers to a substance being carried along with fluid motion. Consider a contaminant being advected downstream with some fluid flowing through a one-dimensional pipe at a constant velocity, $u$. Then the concentration or density $c$ of the contaminant satisfies the advection equation of the form

$$
\frac{\partial c}{\partial t}+u \frac{\partial c}{\partial x}=0
$$

It is easy to verify that this equation admits solutions of the form

$$
c(x, t)=c(x-u t)
$$

for any function $c$. The concentration profile or wave form specified by $C$ simply propagates with constant speed $u$ and unchanged shape. Equation (1) is generally called one space dimension advection equation. Similarly, we can get the two space dimension advection equation

$$
\frac{\partial c}{\partial t}+u \frac{\partial c}{\partial x}+v \frac{\partial c}{\partial y}=0
$$

\footnotetext{
*E-mail address: wuteng@hhu.edu.cn ISSN: 1791-2377@ 2013 Kavala Institute of Technology. All rights reserved.
}

In Equation (3), $\mathrm{u}$ is the flow velocity in the $\mathrm{x}$ direction, $\mathrm{v}$ is the flow velocity in the $\mathrm{y}$ direction, $\mathrm{x}$ and $\mathrm{y}$ are flow directions, and $\mathrm{c}$ is the transport substance concentration. As to 1-D advection equation, the classical up-wind difference scheme can be express as [4],

$$
\begin{aligned}
& c_{j}^{n+1}=c_{j}^{n}-u \Delta t\left(c_{j}^{n}-c_{j-1}^{n}\right) / \Delta x \quad u>0 \\
& c_{j}^{n+1}=c_{j}^{n}-u \Delta t\left(c_{j+1}^{n}-c_{j}^{n}\right) / \Delta x \quad u<0
\end{aligned}
$$

But the accuracy is not quite high, especially in shock capturing $[5,6,7,8]$. The truncation error is so large that its using area is confined. Efforts have been made to obtain a class of schemes for this equation $[9,10,11,12]$. In this paper, a new upwind difference scheme based on the undetermined coefficient method for the advection equations with the dimensionless parameters was developed to reduce the numerical diffusion.

\section{Upwind scheme based on the undetermined coefficient} method

\subsection{The new upwind scheme for 1-D advection equation} Case 1: $\mathrm{u}>0$

When the velocity, parameter $\mathrm{u}$ in Eq.(1), is greater than zero, $c_{j-1}^{n-1}$ and $c_{j}^{n-1}$ can be introduced to the conventional upwind scheme, Eq. (4), and the new upwind scheme is constructed as,

$$
c_{j}^{n+1}=a_{1} c_{j-1}^{n}+a_{2} c_{j}^{n}+a_{3} c_{j-1}^{n-1}+a_{4} c_{j}^{n-1} u>0,
$$


Using Taylor expansion about the point ( $n, j)$, we obtain

$\left(a_{1}+a_{2}+a_{3}+a_{4}-1\right) c_{j}^{n}+\left(-\Delta t-a_{3} \Delta t-a_{4} \Delta t\right) \frac{\partial c}{\partial t}$

$+\left(-a_{1} \Delta x-a_{3} \Delta x\right) \frac{\partial c}{\partial x}+\left(-\frac{1}{2} \Delta t^{2} u^{2}+\frac{1}{2} a_{1} \Delta x^{2}\right.$

$\left.+\frac{1}{2} a_{3}\left(\Delta t^{2} u^{2}+\Delta x^{2}-2 \Delta t \Delta x u\right)+\frac{1}{2} a_{4} \Delta t^{2} u^{2}\right) \frac{\partial^{2} c}{\partial x^{2}}$

$+\left(\frac{1}{6} \Delta t^{3} u^{3}-\frac{1}{6} a_{1} \Delta x^{3}-\frac{1}{6} a_{3}\left(-\Delta t^{3} u^{3}+3 \Delta t^{2} u^{2} \Delta x\right.\right.$

$\left.\left.-3 \Delta t u \Delta x^{2}+\Delta x^{3}\right)+\frac{1}{6} a_{4} \Delta t^{3} u^{3}\right) \frac{\partial^{3} c}{\partial x^{3}}+\ldots=0$

Comparing (7) and (1), we have the following equations.

$$
\begin{aligned}
& a_{1}+a_{2}+a_{3}+a_{4}=1 \\
& a_{1}+\left(1-c_{r}\right) a_{3}-c_{r} a_{4}=c_{r} \\
& a_{1}+\left(c_{r}^{2}-2 c_{r}+1\right) a_{3}+c_{r}^{2} a_{4}=c_{r}^{2} \\
& a_{1}+\left(-c_{r}^{3}+3 c_{r}^{2}-3 c_{r}+1\right) a_{3}-c_{r}^{3} a_{4}=c_{r}^{3}+m
\end{aligned}
$$

where $\mathrm{m}$ is a dimensionless parameter to reduce the numerical diffusion, and $C_{r}=(u \Delta t) / \Delta x$. By solving the Eq. (8)-(11), we get the expressions of the parameters:

$a_{1}=\frac{4 c_{r}^{3}-2 c_{r}^{2}+2 m c_{r}+m}{\left(c_{r}+1\right) c_{r}}$

$a_{2}=1-\frac{4 c_{r}^{3}-c_{r}^{2}+(2 m-1) c_{r}+3 m}{\left(c_{r}+1\right) c_{r}}$

$a_{3}=\frac{2 c_{r}^{3}-2 c_{r}^{2}+m}{c_{r}\left(c_{r}+1\right)}$,

$a_{4}=\frac{-2 c_{r}^{3}+3 c_{r}^{2}-c_{r}+m}{\left(c_{r}+1\right) c_{r}}$,

\section{Case 2: $\mathbf{u}<\mathbf{0}$}

When there is $\mathrm{u}<0, c_{j+1}^{n-1}$ and $c_{j}^{n-1}$ are introduced to Equation (5), and we get

$$
c_{j}^{n+1}=b_{1} c_{j}^{n}+b_{2} c_{j+1}^{n}+b_{3} c_{j}^{n-1}+b_{4} c_{j+1}^{n+1}
$$

At the point (n, j), using Taylor expansion we have

$$
\begin{aligned}
& \left(b_{1}+b_{2}+b_{3}+b_{4}-1\right) c_{j}^{n}+\left(-\Delta t-b_{3} \Delta t-b_{4} \Delta t\right) \frac{\partial c}{\partial t} \\
& +\left(b_{2} \Delta x+b_{4} \Delta x\right) \frac{\partial c}{\partial x}+\left(-\frac{1}{2} \Delta t^{2} u^{2}+\frac{1}{2} b_{2} \Delta x^{2}\right. \\
& \left.+\frac{1}{2} b_{4}\left(\Delta t^{2} u^{2}+\Delta x^{2}+2 \Delta t \Delta x u\right)+\frac{1}{2} b_{3} \Delta t^{2} u^{2}\right) \frac{\partial^{2} c}{\partial x^{2}} \\
& \frac{1}{6} \Delta t^{3} u^{3}-\frac{1}{6} b_{2} \Delta x^{3}-\frac{1}{6} b_{4}\left(\Delta t^{3} u^{3}+3 \Delta t^{2} u^{2} \Delta x\right. \\
& \left.\left.+3 \Delta t u \Delta x^{2}+\Delta x^{3}\right)+\frac{1}{6} b_{3} \Delta t^{3} u^{3}\right) \frac{\partial^{3} c}{\partial x^{3}}+\ldots=0
\end{aligned}
$$

Substituting (1) into (13), we obtain

$$
b_{1}+b_{2}+b_{3}+b_{4}=1
$$

$$
b_{2}+b_{4}+\left(b_{3}+b_{4}+1\right) c_{r}=0
$$

$-c_{r}^{2}+c_{r}^{2} b_{3}+b_{2}+b_{4}\left(c_{r}^{2}+2 c_{r}+1\right)=0$

$$
c_{r}^{3}+c_{r}^{3} b_{3}+b_{2}+b_{4}\left(c_{r}^{3}+3 c_{r}^{2}+3 c_{r}+1\right)=n
$$

where $\mathrm{n}$ is a dimensionless parameter. Solving Eqs (13)(17), we have

$$
b_{1}=1-b_{2}-b_{3}-b_{4}
$$

$b_{2}=\frac{4 c_{r}^{2}+2 c_{r}}{1-c_{r}}+\frac{c_{r}^{2}-c_{r}+1}{c_{r}\left(c_{r}-1\right)} n$

$b_{3}=\frac{2 c_{r}^{2}+3 c_{r}+1-n}{c_{r}-1}$

$b_{4}=\frac{-2 c_{r}^{2}\left(c_{r}+1\right)+n}{c_{r}\left(1+c_{r}\right)}$

Unifying case 1 and case 2, we obtain the new upwind scheme method of the form

$c_{j}^{n+1}=A_{1} c_{j-1}^{n}+A_{2} c_{j}^{n}+A_{3} c_{j+1}^{n}+A_{4} c_{j-1}^{n-1}+A_{5} c_{j}^{n-1}+A_{6} c_{j+1}^{n-1}$

Where

$A_{1}=\frac{u+|u|}{2 u} a_{1}$

$A_{2}=\frac{u+|u|}{2 u} a_{2}+\frac{u-|u|}{2 u} b_{1}$

$A_{3}=\frac{u-|u|}{2 u} b_{2}$ 


$$
\begin{aligned}
& A_{4}=\frac{u+|u|}{2 u} a_{3} \\
& A_{5}=\frac{u+|u|}{2 u} a_{4}+\frac{u-|u|}{2 u} b_{3} ; \\
& A_{6}=\frac{u-|u|}{2 u} b_{4}
\end{aligned}
$$

Formula (18) is the new upwind scheme based on the undetermined coefficient method for 1-D advection equation.

\subsection{The new upwind scheme for 2-D advection equation}

Vectors split method is employed in the numerical solution of the 2-D advection equation [2]. The two-dimensional convection is expressed in the following two equations, Eq, (19) and Eq. (20).

$$
\begin{aligned}
& \frac{\partial c}{\partial t}+u \frac{\partial c}{\partial x}=0 \\
& \frac{\partial c}{\partial t}+v \frac{\partial c}{\partial y}=0
\end{aligned}
$$

Using the undetermined coefficients method, we can get the numerical solutions of the above equations.

$$
\begin{aligned}
c_{i}^{n+1 / 2}= & C_{1} c_{i-1}^{n}+C_{2} c_{i}^{n}+C_{3} c_{i+1}^{n} \\
& +C_{4} c_{i-1}^{n-1}+C_{5} c_{i}^{n-1}+C_{6} c_{i+1}^{n-1}, \\
c_{i}^{n+1}= & D_{1} c_{i-1}^{n+1 / 2}+D_{2} c_{i}^{n+1 / 2}+D_{3} c_{i+1}^{n+1 / 2} \\
& +D_{4} c_{i-1}^{n-1 / 2}+D_{5} c_{i}^{n-1 / 2}+D_{6} c_{i+1}^{n-1 / 2}
\end{aligned}
$$

Same as (18), it is easy to get the expression of parameters in (21) and (22).

\section{Numerical experiments}

When the velocity $u$ is constant, Equation (1) has analytical solution. For the original boundary conditions of $C(x, 0)=f(x),-\infty<x<\infty$, the analytical solution of Equation (1) is

$$
C(x, 0)=f(x-u t)
$$

(1) Case of one-dimensional rectangular wave Set the initial concentration as

$$
\begin{aligned}
& C(x, t)=1, \quad x_{0} \leq x \leq x_{1} \\
& C(x, t)=0, \quad-\infty \leq x<x_{0}, \quad x_{1}<x \leq+\infty
\end{aligned}
$$

Computing parameters: (1) $u>0, x_{0}=150, x_{1}=350$, $\Delta x=10, \Delta t=10 \mathrm{~s}, u=0.4 \mathrm{~m} / \mathrm{s}, m=0.02$, and the total computing time is 1000 seconds; (2) $u<0$, $x_{0}=950, x_{1}=1150, \Delta x=10, \Delta t=10 \mathrm{~s}$, $u=-0.4 \mathrm{~m} / \mathrm{s}, n=0.02$, the total computing time is 1000 seconds. The computed results are shown in Figure 1. It indicates that, whether $\mathrm{u}>0$ or $\mathrm{u}<0$, the computed results of the new scheme based on the undetermined coefficient method are close to the analytical solution results.

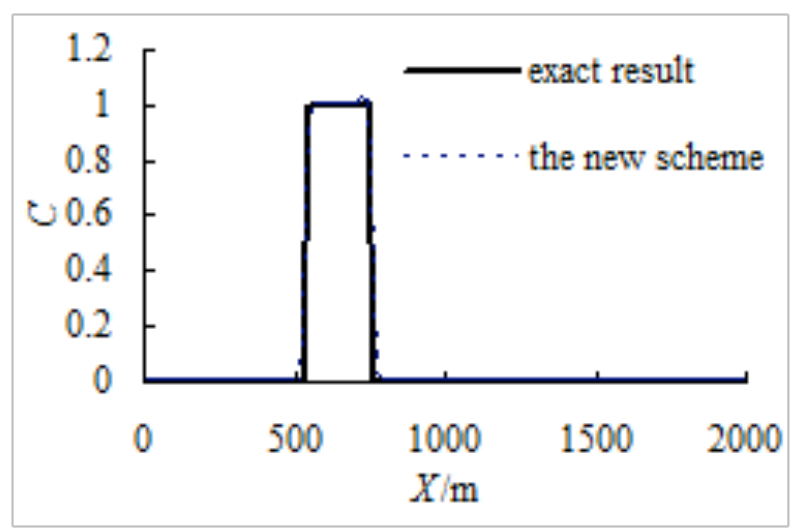

(a) $\mathrm{u}>0$

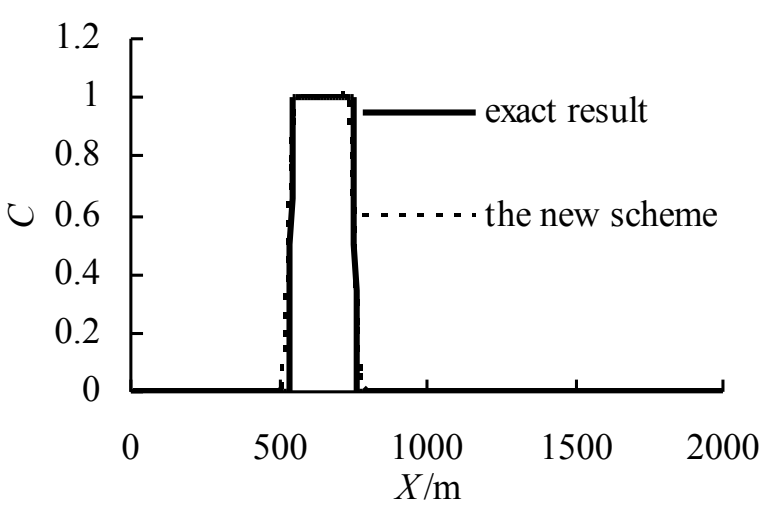

(b) $\mathrm{u}<0$

Fig.1. Test on the rectangular wave with the conventional upwind scheme and the new method

(2) Case of two-dimensional Gauss wave

When the velocity in two-dimensional convection equation is constant, the initial surface will move horizontally over time. Set the initial surface expressed as

$$
c(x, y)=\exp \left[-\frac{\left(x-x_{0}\right)^{2}}{2 \delta_{0}{ }^{2}}-\frac{\left(y-y_{0}\right)^{2}}{2 \delta_{0}{ }^{2}}\right],
$$

where $u=-0.4 \mathrm{~m} / \mathrm{s}, \quad v=-0.4 \mathrm{~m} / \mathrm{s}, \quad x_{0}=1200$, $y_{0}=1200, \delta_{0}=100, \quad t=10 \mathrm{~s}, \quad x=10 \mathrm{~m}, \quad y=10 \mathrm{~m}$, the total computing time is 800 seconds, and the original conditions $c(0, y, t)=0, c(x, 0, t)=0$. The computed results are shown in Figure 2. It showed that the Gaussian wave shape was kept well after 800 seconds, and the numerical diffusion is little. The new upwind scheme can be used to compute the advection of two-dimensional Gauss wave. 


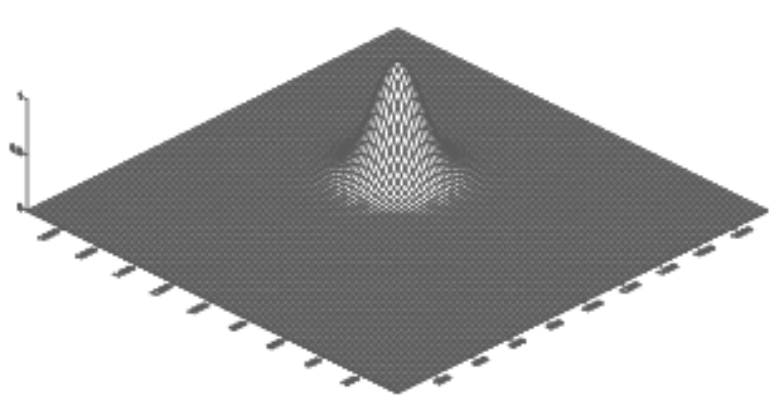

(a) initial value

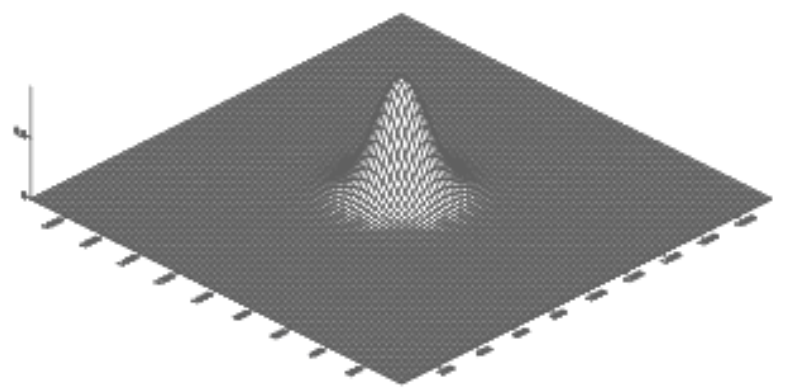

(b) Computed value

Fig. 2. Test on the 2-D Gaussian wave by the new upwind scheme

(3) Case of two-dimensional rectangular wave

Most schemes can well simulate waves with flat changes, such as Gauss wave and elliptical wave, etc. But when the wave changes rapidly such as rectangular wave, it is hard to get good simulation results, and serious dispersion and dissipation problems generally occur. To verify the performance of the new upwind scheme in solving twodimensional advection equation, test on the new upwind scheme is deployed. In this case, the height of the rectangular wave is 1.0 , the top width and length are $200 \mathrm{~m}$, and the rectangular wave locates at $750 \leq x \leq 10500$ and $750 \leq \mathrm{y} \leq 1050$ at the beginning. In the computation, the parameters set as $\Delta \mathrm{t}=5 \mathrm{~s}, \Delta \mathrm{x}=10 \mathrm{~m}, \Delta \mathrm{y}=10 \mathrm{~m}, \mathrm{u} 0=-0.4 \mathrm{~m} / \mathrm{s}$, $\mathrm{v} 0=-0.4 \mathrm{~m} / \mathrm{s}$, and $\mathrm{t}=500 \mathrm{~s}$. The plane extent is $0 \sim 2000 \mathrm{~m}$ respectively in $\mathrm{x}$ and $\mathrm{y}$ direction. When $\mathrm{u}$ and $\mathrm{v}$ are constants, Equation (1) has analytical solution. Let $\mathrm{s}(\mathrm{x}, \mathrm{y}, 0)=\mathrm{f}(\mathrm{x}, \mathrm{y})$, and the analytical solution of Equation (1) on any time $t$ can be expressed as

$$
s(x, y, t)=f(x-u, y-v, t),
$$

which means that the initial wave shape moves horizontally without numerical diffusion after $\mathrm{t}$ seconds later, and the moving distances are ut meter and vt meter respectively in $\mathrm{x}$ and $\mathrm{y}$ direction. Figure 3 is the computing result on the 2-D rectangular wave by the new upwind scheme. It showed that the new upwind scheme had a little numerical diffusion after 800 seconds later, while it can reflect the rectangular wave shape well. It presents a good result in solving $2-\mathrm{D}$ rectangular wave problem.

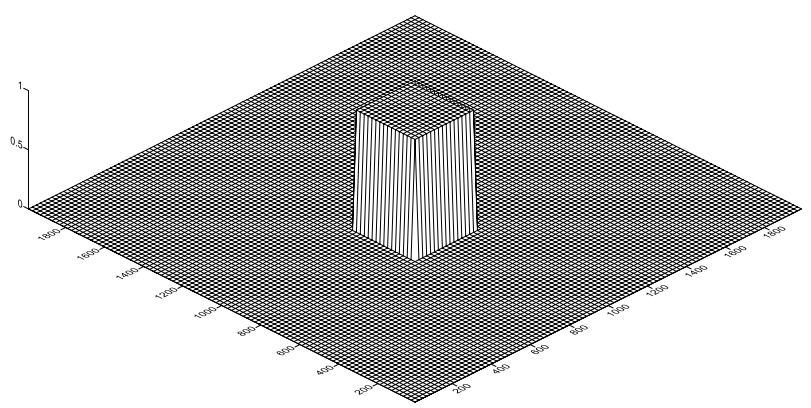

(a) initial value

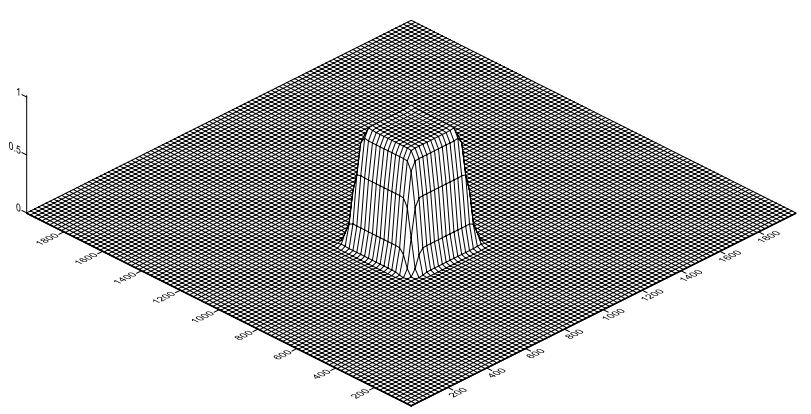

(b)Computed value

Fig. 3. Test on the 2-D rectangular wave by the new upwind scheme

\section{Conclusions}

Numerical diffusion generally occurs in the conventional upwind scheme when it is applied to resolve advection equations. To reduce the numerical diffusion, a highresolution is constructed based on the undetermined coefficient method. In the test of rectangular wave and Gauss wave, the results of the new scheme method are agreed with the exact solution. The new scheme has better accuracy than the conventional upwind scheme in computing the rectangular wave.

\section{Acknowledgement}

The work is supported by the National Science Foundation of China (51039003).

\section{References}

1. Holly, F. M. Jr.; Preissmann, A., "Accurate calculation of transport in two dimensions". Journal of Hydraulic division. ASCE. 103(11), 1977,pp.1259-1277.

2. Steger, J. L.; Warming, R. F., "Flux splitting of inviscid gas dynamics equations with application to finite-difference methods". J. Computational physics, 40, 1981, pp263-293.

3. Shu, C.W.; Osher, S., "Efficient implementation of essentially nonoscillatory shock-capturing schemes". J. Computational physics, 83,1989, pp 32-78.

4. Osher, S., "Upwind difference schemes for hyperbolic systems of conservation laws". Mathematics of computation, 38,1982, pp339374.
5. Yamileva, A.M.; Medvedev, A.Yu. ; Nasibullayev, I.Sh.; Selivanov, A.S.; Gazizov, R.K.; Vairis, A.; "A two-parameter 2D-model of the elastic stage of linear friction welding using ANSYS mechanical finite element analysis programme". Journal of Engineering Science and Technology Review, 5(3), 2012, pp 6-9.

6. Wenya Li, Shanxiang Shi, Feifan Wang, Zhihan Zhang, Tiejun Ma, Jinglong $\mathrm{Li}$, Numerical simulation of friction welding processes based on ABAQUS environment. Journal of Engineering Science and Technology Review, 2012 5(3):10-19

7. Vairis, A. Mathematical modelling of the linear friction welding process. Journal of Engineering Science and Technology Review, 5(3) ,2012, pp25-31. 
8. Bakhti, F.Z.; Si-Ameur, M., "Numerical simulation of mixed convection in a inclined thick duct". Journal of Engineering Science and Technology Review, 4(2), 2011, pp 152-159.

9. Teng, Wu.; Hongwu, Zhang. Deyu, Zhong., "Up-wind scheme based on the undetermined coefficient method". J Tsinghua Univ (Sci \&Tech), 49(12), 2009, pp154-157. (in Chinese)

10. Zuotao, Xie.; Xiaofeng, Zhang., "Improvement of HollyPreissmann scheme. Hydro science and engineering”. 1, 2002, pp12-18. (in Chinese)

11. Xiaofeng, Zhang.; Hongwu, Zhang., "Efficient improvement of Crank-Nicolson scheme". Advance in water science.12(1), 2001, pp33-38. (in Chinese)

12. Xiaofeng, Zhang.; Jun, Chen., "Improvement of Leap-frog scheme". Engineering journal of Wuhan university, 38(5), 2005, pp6-10. 